\title{
ENTEROBACTER SPECIES CAUSING MENINGITIS IN PATIENT ADMITTED WITH FEBRILE ENCEPHALOPATHY
}

\author{
Shadma Yaqoob, Priyanka Tripathi, Zahida Parveen Dar, Y.Ibotomba Singh
}

1. Assistant Professor, Department of Microbiology, Era's Lucknow Medical College and Hospital. Lucknow.

2. Lecturer, Department of Microbiology, Era's Lucknow Medical College and Hospital. Lucknow.

3. Junior Resident, Department of Microbiology, Era's Lucknow Medical College and Hospital. Lucknow.

4. Professor \& Head, Department of Microbiology, Era's Lucknow Medical College and Hospital. Lucknow.

\section{CORRESPONDING AUTHOR}

Dr. Shadma Yaqoob

$20 / 253$

Indra Nagar, Lucknow

E-mail: drshadmayaqoob@yahoo.com

Ph: 00919415521967.

ABSTRACT: Enterobacter species are increasingly a cause of nosocomial meningitis among neurosurgery patients, but risk factors for these infections are not well defined. It was found that external cerebrospinal fluid (CSF) drainage devices, isolation of Enterobacter species from a non-CSF culture, prolonged administration of antimicrobial drugs before the diagnosis of meningitis that were inactive in vitro against Enterobacter species, immunosuppression and advanced age are also independent risk factors for Enterobacter Meningitis. Despite favourable treatment outcomes, EM is a serious infection associated with Enterobacter species colonization or infection at other surgical sites, with selective antimicrobial pressure, and with invasive CNS devices. The treatment is difficult because of inability of many of the antibiotics that are active against these bacteria to achieve adequate concentration in CSF. However third generation cephalosporins are active against gram negative bacilli and also achieved high concentration in CSF.

KEY WORDS: Enterobacter, Febrile Encephalopathy, Meningitis

INTRODUCTION: Gram negative bacilli rarely cause Central nervous system (CSF) infections in adults but they have become increasingly common in patients with a history of impaired host defences resulting from iatrogenic contamination of the cerebrospinal fluid, either directly during surgery, or indirectly by creating a defect through an infection defence barrier, providing a route of entry for organisms that invade the meninges ${ }^{1,2}$. Appropriate empirical antimicrobial therapy for the treatment of gram-negative bacillary meningitis is essential to prevent morbidity and mortality

CASE REPORT: A 45 year old male was admitted with low grade fever for past 15 days, vomiting and drowsiness for 5 days with no history of trauma or abscess .The patient was taking drugs since 20 days with no description of drugs available. On examination meningeal irritation was present with reflexes increased on right side. On palpation no organomegaly present. Patient was taken to Radiology Dept for MRI that revealed the presence of tuberculoma. The clinician was treating the patients with levofloxacin, mannitol and antitubercular drugs but the patient was not responding. The fever was increased from $100^{\circ} \mathrm{F}$ to $103^{\circ} \mathrm{F}$ along with neck stiffness. On day ten, a spinal tap was performed. . A lumbar puncture is done by positioning the person, usually lying on the side, applying local anaesthetic, and inserting a needle into the dural sac to collect cerebrospinal fluid (CSF). It revealed cloudy 
cerebrospinal fluid, with a profile consistent with pyogenic meningitis. The pressure was elevated. The CSF sample was examined for presence and types of white blood cells, red blood cells, protein content and glucose level. Glucose level was decreased, protein content was high \& polymorphonuclear neutrophils was more than 300/ $\mathrm{mm}^{3} \cdot \mathrm{In}$ Gram staining of the sample bacteria was absent ( this does not exclude bacterial meningitis as they are only seen in $60 \%$ of cases; this figure is reduced by a further $20 \%$ if antibiotics were administered before the sample was taken).

Microbiological culture of the sample was done (it identifies the organism in 70-85\% of cases). Identification and speciation was done on the basis of motility, gram staining and biochemical tests. It was motile, produced gas, Indole and Methy Red negative, Voges proskauer and citrate positive, decarboxylated Arginine \& Lysine.

Antibiotic sensitivity test was done by Kirby Bauer methods and then clinician started the treatment with Cefipime 2gm every 8hrs I/V for 28 days and Gentamycin $5 \mathrm{mg}$ every $24 \mathrm{hrs}$ I/V for 6 days with mannitol and antitubercular drugs. Patient responded well with the above treatment. After 1 month of treatment he was switched over to oral drugs and discharged.

DISCUSSION: Enterobacter species are increasingly as the cause of nosocomial meningitis and this has been documented following disruption of the dura-arachnoid barrier secondary to trauma, impaired host defences or surgery ${ }^{3,4}$. Risk factors for these types of infections are not clearly defined but some of the studies indicate external CSF drainage devices, prolonged administration of antimicrobial drugs prior to the diagnosis of meningitis, isolation of Enterobacter from a non-CSF culture, immunosuppression as independent risk factors for Enterobacter meningitis 5 .

This case report provides clinical evidence to support administration of I/V cefipime \& gentamycin in critically ill adult patients with Enterobacter meningitis that are active against gram negative bacilli and also achieved high concentration in CSF.

A good antibiotic policy should be laid down between the clinician and microbiologist in all tertiary care hospitals and a strict antibiotic regimen should be applied by clinicians. Those patients identified with history of chronic illness like diabetes mellitus, tuberculosis, renal failure, perito-neal dialysis should be dealt with utmost care.

ACKNOWLEDGEMENT: I would like to express my sincere thanks to Dr. Fareya Haider, junior resident, Microbiology Department for her timely help during the study. She provided me all the clinical details of the patient when needed.

\section{REFERENCES:}

1. Durand ML, Caderwood SB, et al. Acute Bacterial Meningitis in adult. a review of 493 episodes. N Engl J Med 1993;328:21-8.

2. Berk SL, McCabe WR. Meningitis caused by Gram-negative bacilli. Ann Intern Med 1980;93:253-60.

3. Gower DJ, Barrows AS, Kelly D.L, et al. Gram-negative bacillary meningitis in the adult: a review of 39 cases. South Med J 1986;79:1499-502.

4. Huang CR, Lu CH, Chang WN Adult Enterobacter meningitis. a high incidence of coinfection with other pathogens and frequent association with neurosurgical procedures. Infection 2001;29(2):75-9. 
5. Parodi S, Lechner A, Osih R, et al. Nosocomial Enterobacter meningitis. Risk factors, management, and treatment outcomes. CID 2003;37:159-66.

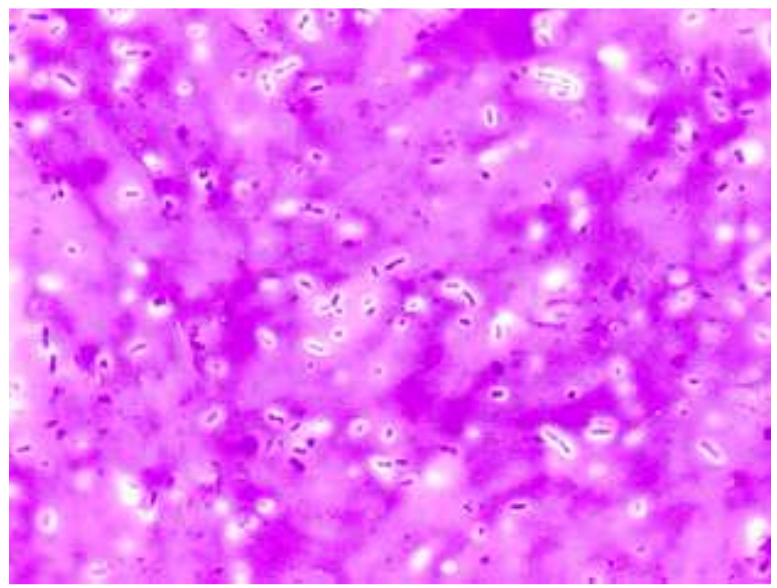

Fig-1 Gram Negative Bacilli-100x

Fig-2 Methyl Red test
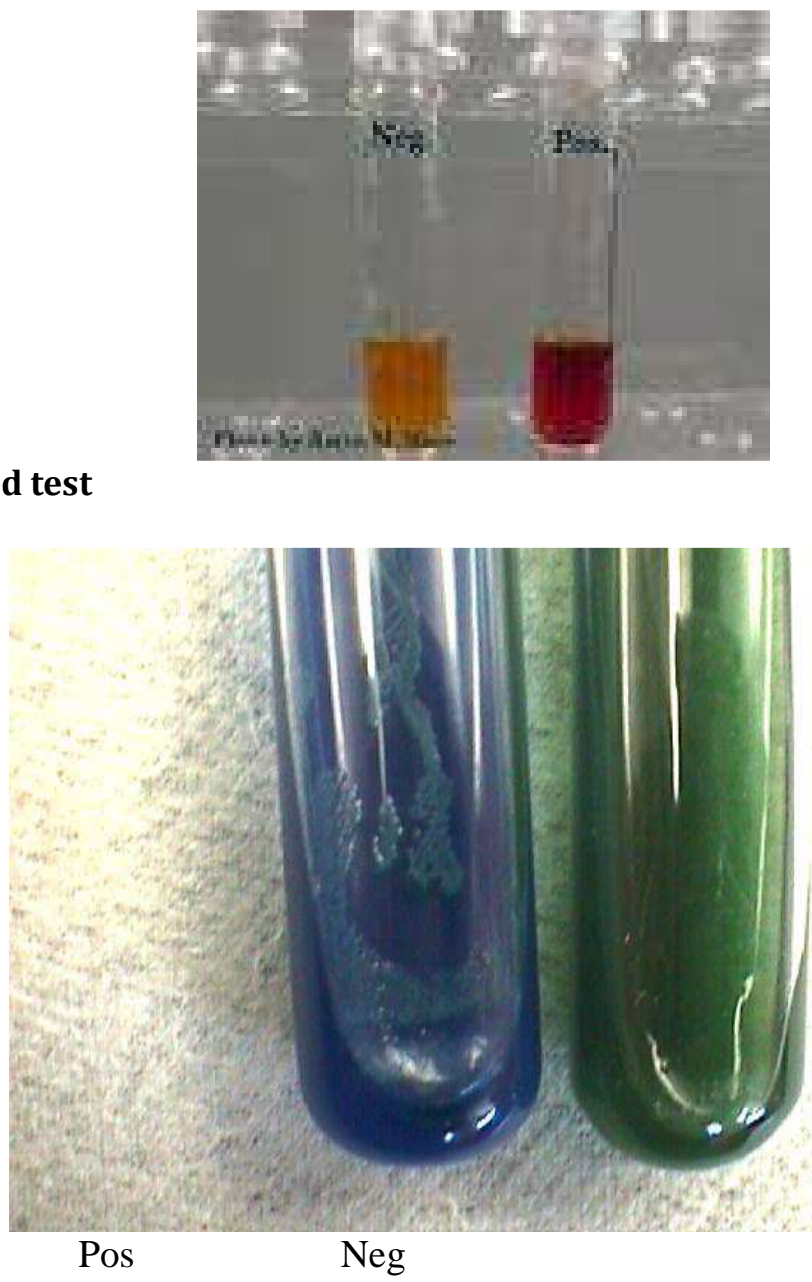

Fig-3 Citrate utilisation test 